\title{
Automation Countermeasure System for Intersection Optimization
}

\author{
Hua-pu Lu, ${ }^{1}$ Zhi-yuan Sun, ${ }^{1}$ Wen-cong Qu, ${ }^{1}$ and Yue $\mathrm{Li}^{1,2}$ \\ ${ }^{1}$ Institute of Transportation Engineering, Tsinghua University, Beijing 100084, China \\ ${ }^{2}$ Transport Planning and Research Institute, Ministry of Transport, Beijing 100028, China \\ Correspondence should be addressed to Yue Li; liu_liu_30@sina.com
}

Received 4 December 2014; Accepted 24 December 2014

Academic Editor: Qingang Xiong

Copyright (c) 2015 Hua-pu Lu et al. This is an open access article distributed under the Creative Commons Attribution License, which permits unrestricted use, distribution, and reproduction in any medium, provided the original work is properly cited.

\begin{abstract}
To satisfy the demand of congestion problem solving in intersections, this paper studies the method of automation countermeasure system for intersection optimization (ACSIO). Taking into account the extensive contents and objectives of intersection optimization, this paper puts forward the functions and architecture of ACSIO based on intersection optimization problem statement. Seeking optimal design of intersection channelization and signal control, the main goal of ACSIO is to achieve dynamic and coordination management of intersection. The problem is formulated as a multiobjective program, with each objective corresponding to a different player in the system. Moreover, it presents system design of ACSIO. A case study based on a realworld intersection is implemented to test the efficiency and applicability of the proposed modeling and computing methods.
\end{abstract}

\section{Introduction}

Traffic congestion is getting increasingly serious in the urbanized advancement. Beijing, China, can be treated as an example of megalopolis. By the end of 2013, permanent residents was 21.14 million and vehicle possession was 0.54 million [1]. According to "Beijing Road Traffic Operation Analysis Report (2014.5)," traffic performance index of working day in May was 5.8, an increase of $5.5 \%$ over the same period last year [2]. Intersection traffic problem is one of the main reasons of traffic congestion [3]. From 2011 to 2013, Beijing Traffic Management Bureau conducted the "Third-Round Project of Beijing Intelligent Traffic Management" to solve traffic problems of 330 intersections [4]. It has very important significance to optimize the intersection traffic organization.

The contents of intersection optimization can spread from many aspects, such as channelization [5], single intersection control [6], green wave control [7], regional coordination control [8], combinatorial optimization [9], road traffic engineering design [10], transit signal priority control [11], pedestrians and bicycles [12], environmental effect [13], and traffic safety [14]. Intersection optimization is generally implemented based on bilevel programming model [15] or multiobjective programming model [16]. The objectives of intersection optimization are extremely diverse, namely, delay [17], stops or stop rate [18], queue length [19], capacity or saturation [20], vehicle emission [21], energy consumption [22], and conflict point [23].

As shown above, intersection optimization involves extensive contents and objectives. Moreover, for cities, especially the mega cities, the number of intersections is very great. Accordingly, the workload of intersection optimization is extremely heavy. Considering relatively small staff size of traffic management and scientific institution, it is very necessary to establish automation countermeasure system for intersection optimization. However, taking into account all the present researches in this field, there are some limitations. Current study mainly focused on traffic signal control, and channelization is designed based on engineering experience and human-computer interaction [24]. In addition, feasibility analysis of automation countermeasure system is needed for the extensive contents, and application analysis of optimization objectives is needed for the extensive objectives.

In this paper, the method of automation countermeasure for intersection optimization based on multiobjective programming model, which perceives ability of optimal design of 
intersection channelization and signal control, is put forward in detail. The remainder of this paper is organized as follows. Section 2 presents intersection optimization problems. In Section 3, the methodology of automation countermeasure system for intersection optimization is put forward. In Section 4, automation countermeasure system design is present. A case study based on a real-world road network is carried out in Section 5 to demonstrate the performance and applicability of the proposed method. Finally, conclusions are drawn in Section 6.

\section{Intersection Optimization Problem Statement}

2.1. Feasibility Analysis of Automation Countermeasure. It is necessary to perform feasibility analysis of automation countermeasure because of the extensive contents of intersection optimization. Feasibility analysis, based on qualitative analysis (expert appraisal and engineering experience), is implemented in the following aspects [25]:
(a) intention and significance;
(b) application area;
(c) technical principle;
(d) automation requirements.

Then, conclusions are drawn, as shown in Table 1.

2.2. Application Analysis of Optimization Objectives. At present, urban traffic problems are mainly reflected in the following aspects.

(a) Traffic Congestion. According to "2013 Beijing Transport Annual Report" [26], the total number of trips (excluding walking) in Beijing (within Sixth Ring) in 2012 was 30.33 million per day, an increase of 5.6 per cent over the previous year, and the traffic network load in peak hours increased by $4.4 \%$. To improve the traffic efficiency is one of the most important means to solve the problems of traffic congestion.

(b) Environmental Pollution. According to "2013 China Environment Condition Communique" [27], the national average duration of the haze in 2013 was 35.9 days, an increase of 18.3 days over the previous year. Considering traffic emission is one of the reasons of the haze, environmental effect is an important question that should be solved in urban traffic management.

(c) Accident Threat. According to "2013 China Road Traffic Accident Statistics Annual Report” [28], the number of traffic accidents involving death or injury in 2013 was 0.198 million people, and direct property damage was 10.4 billion yuan. Since traffic accident causes heavy casualties and reduces traffic capacity, traffic safety is a factor that must be considered.

In summary, traffic efficiency, environmental effect, and accident threat are the important considerations of intersection optimization. Therefore, the intersection optimization objectives are shown in Table 2.
TABLE 1: Automation countermeasure for intersection optimization.

\begin{tabular}{lcc}
\hline Number & Countermeasure & Content \\
\hline 1 & Channelization & $\begin{array}{c}\text { Lane and sharing } \\
\text { Lane width }\end{array}$ \\
\hline 2 & Single control & $\begin{array}{c}\text { Phase setting } \\
\text { Time setting }\end{array}$ \\
\hline 3 & Transit signal priority control & $\begin{array}{c}\text { Phase setting } \\
\text { Time setting }\end{array}$ \\
\hline 4 & Road traffic engineering design & Lane and sharing \\
\hline 5 & Pedestrians and bicycles & $\begin{array}{c}\text { Phase setting } \\
\text { Time setting }\end{array}$ \\
\hline 6 & Traffic safety & Phase setting \\
\hline 7 & Environmental effect & $\begin{array}{c}\text { Vehicle emission } \\
\text { Energy consumption }\end{array}$ \\
\hline
\end{tabular}

TABLE 2: Objectives of intersection optimization.

\begin{tabular}{lcc}
\hline Number & Objectives & Indexes \\
\hline 1 & Traffic efficiency & $\begin{array}{c}\text { Managerial effectiveness } \\
\text { Travel feeling }\end{array}$ \\
\hline 2 & Environmental effect & $\begin{array}{c}\text { Vehicle emission } \\
\text { Energy consumption }\end{array}$ \\
\hline 3 & Traffic safety & $\begin{array}{c}\text { Conflict point } \\
\text { Min green time }\end{array}$ \\
\hline
\end{tabular}

2.3. Semantic Coding and Notation Definitions. Since the realization of automation countermeasure is with computerbased learning, semantic coding of intersection is necessary. Semantic coding provides the ability to convert the physical space information of intersection to a symbol system, which facilitates the data identification and processing for computer. Li et al. present the urban road basic geographic elements coding methodology [29]. Li et al. put forward a method of semantic coding of sensor network nodes for road traffic [30]. Physical space information of intersection can be divided into three parts.

(a) Static Data. It is the quantitative description of the geometric design of intersection, including lanes and sharing and lane width (as shown in Figure 1).

(b) Dynamic Data. It describes the dynamic traffic supply and demand of intersection, including signal control scheme and motorcycle type and its volume.

(c) Evaluation Data. It describes the traffic state of intersection, including capacity, delay, vehicle emission, and energy consumption.

Based on semantic coding, the notations used in this paper are described in Notations section.

\subsection{Automation Countermeasure System Organization}

2.4.1. System Functions. The description of system functions is shown in two aspects.

(a) Contents of Intersection Optimization. Based on feasibility analysis of automation countermeasure, the extensive contents of intersection optimization can be simplified to two 


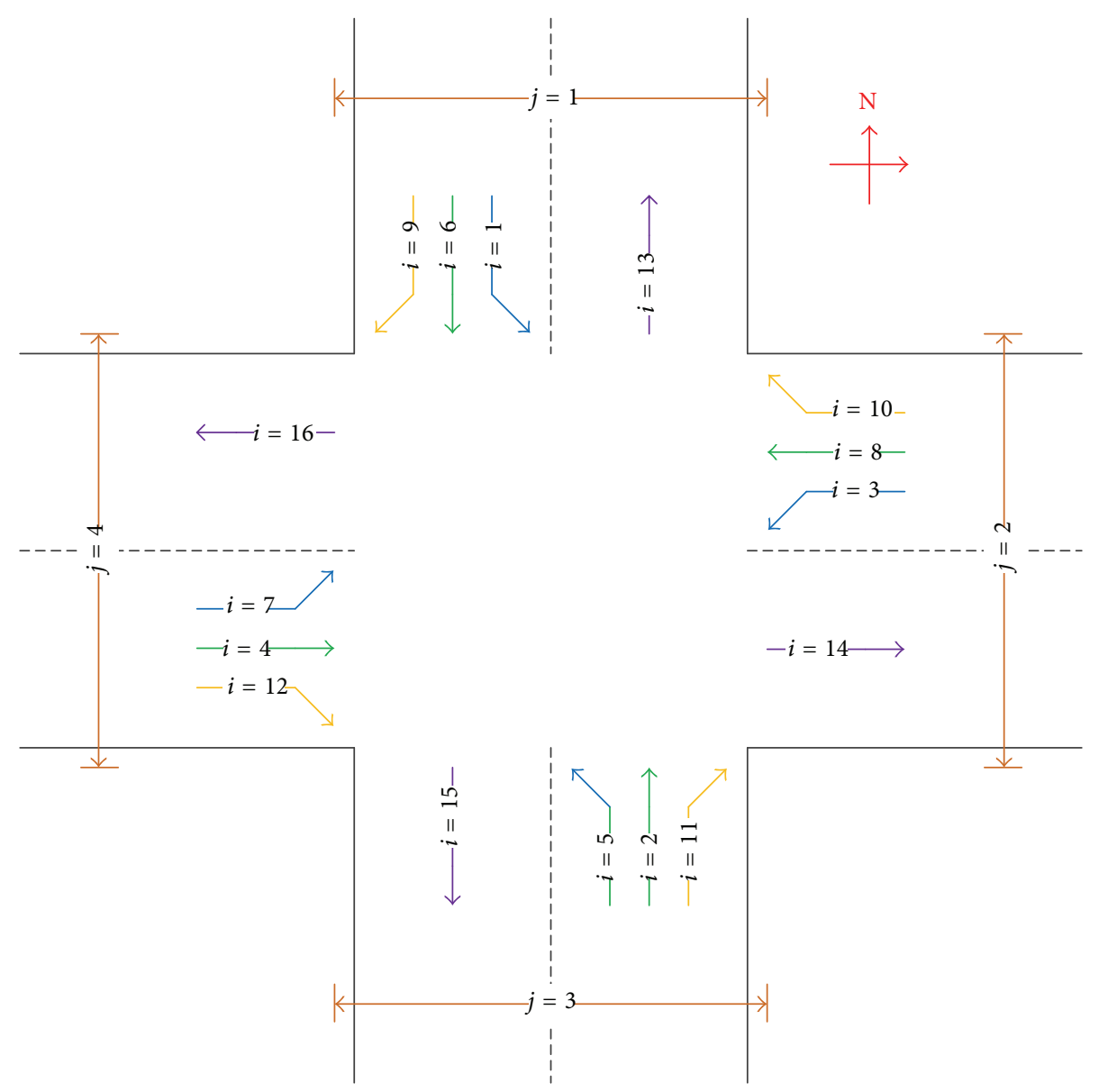

FIGURE 1: Semantic coding of approach and group.

areas, channelization and signal control. The main work of channelization is to design lane and sharing and lane width, considering physical space restrictions of intersection and optimization objectives. The main work of signal control is to design phase setting and time setting, considering traffic safety guarantees of intersection and optimization objectives. Overall consideration of channelization and signal control holds obvious advantages, namely,

(i) high coordination between channelization and signal control;

(ii) dynamic management of channelization and signal control, for example, lane use control for tide traffic.

(b) Objectives of Intersection Optimization. Based on application analysis of optimization objectives, the extensive objectives of intersection optimization can be summarized to three aspects, namely, traffic efficiency, environmental effect, and traffic safety. Since traffic safety is a necessary guarantee objective for all cities, the conflict point and the minimum green time of intersection are treated as restrictive conditions. The concern degree of traffic efficiency and environmental effect for different cities in different periods are even different. In order to ensure the widespread application of the automation countermeasure system, optimization objective selection is operable in the proposed system. Specifically, the weights of different optimization objectives are adjustable. In addition, the recommended method of multiobjective programming is given. Multiobjective programming model holds obvious advantages, namely,

(i) strong pertinence optimization for traffic problems;

(ii) adjustable weight for different optimization objectives according to the actual demand.

2.4.2. System Architecture. According to system requirements and system functions, the system architecture, with four-level architecture, is shown in Figure 2.

(a) Data Resource Level. The data resource level provides the automation countermeasure system with different data from various resources, including existing urban traffic detection systems and investigation. The data types consist of static data and dynamic data.

(b) Actuality Evaluation Level. The actuality evaluation level gives the evaluation of intersection traffic state in three areas. Evaluation indexes include saturation, delay, vehicle 


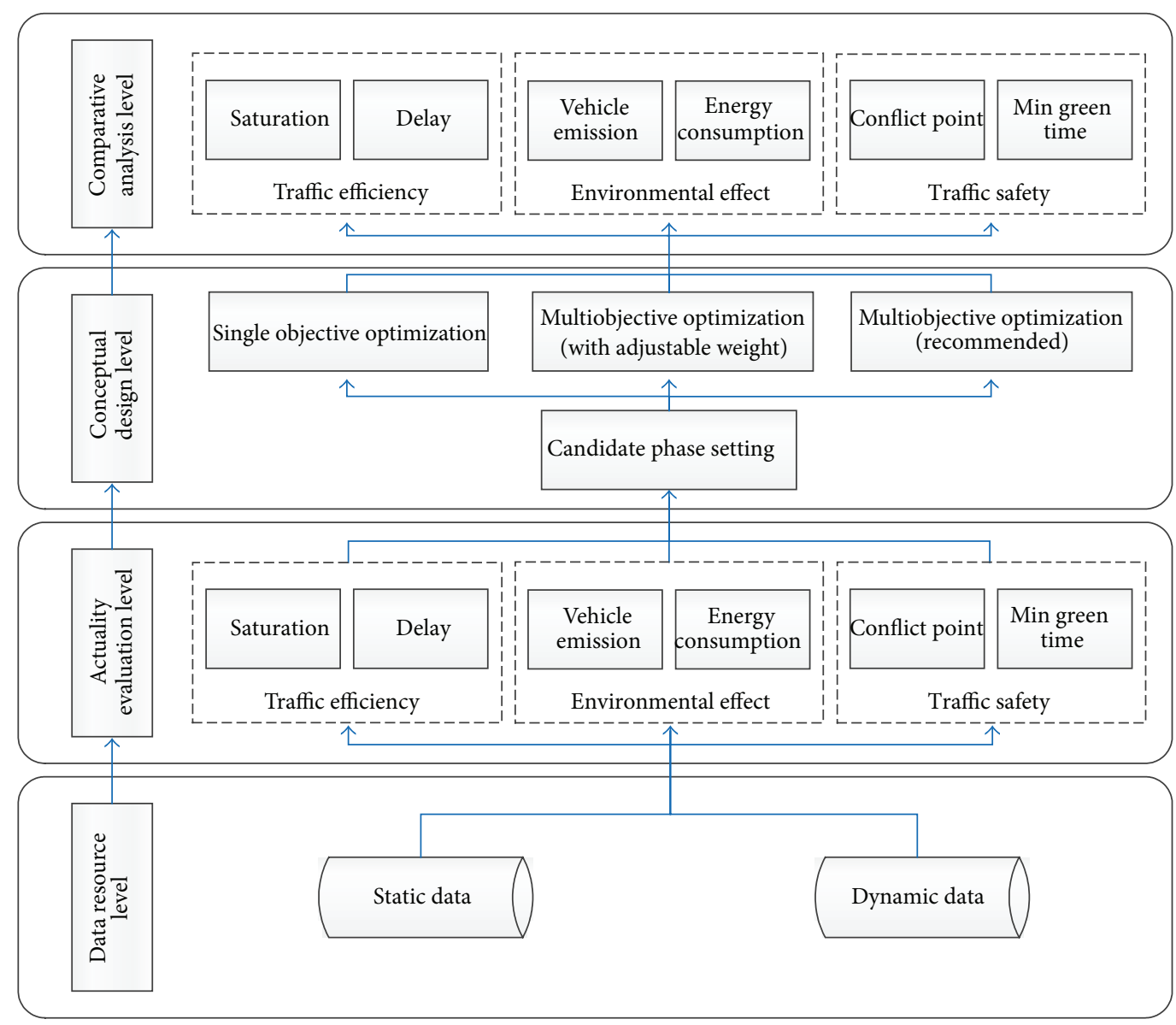

FIGURE 2: Architecture of automation countermeasure system.

emission, energy consumption, conflict point, and minimum green time.

(c) Conceptual Design Level. The conceptual design level is the main part of automation countermeasure system. Considering traffic safety of intersection, especially conflict point, candidate phase setting is proposed based on evaluation of intersection traffic state. Then, the conceptual design level offers three kinds of optimization scheme design methods, including single objective optimization, multiobjective optimization with adjustable weight, and recommended multiobjective optimization.

(d) Comparative Analysis Level. The comparative analysis level is designed to compare status quo scheme with optimization scheme based on several evaluation indexes, the same with actuality evaluation level.

\section{Methodology}

3.1. Traffic Efficiency. The optimization objective of traffic efficiency aims at managerial effectiveness (ME) and travel feeling (TF) improvement. The traditional optimization is to minimize the delay of intersection and maximize the capacity of intersection. (a) Managerial Effectiveness. Capacity is used to evaluate managerial effectiveness. Current research of capacity is relatively mature. The capacity for lane group $i$ is stated as shown [31]:

$$
c_{i}=s_{i} \lambda_{i}=s_{i} \frac{g_{i}}{C} .
$$

The capacity of intersection is formulated as

$$
c_{\text {intersection }}=\sum c_{i}=\sum s_{i} \frac{g_{i}}{C} .
$$

The saturation for lane group $i$ is stated as shown [31]:

$$
X_{i}=\left(\frac{q}{c}\right)_{i}=\frac{q_{i}}{s_{i} \lambda_{i}}=\frac{q_{i} C}{s_{i} g_{i}} .
$$

The capacity balance of different lane groups should be considered in the optimization. That is, the saturation of different lane groups should be basically identical. Consider

$$
\sum\left(\bar{X}_{i}-X_{i}\right)^{2} \leq X_{0}
$$


(b) Travel Feeling. Delay is used to evaluate travel feeling. Current research of capacity is also relatively mature. The delay for lane group $i$ is stated as shown [32]:

$$
d_{i}=\frac{C\left(1-\lambda_{i}\right)^{2}}{2\left(1-\lambda_{i} X_{i}\right)}+\frac{X_{i}^{2}}{2 q_{i}\left(1-X_{i}\right)}-0.65\left(\frac{C}{q_{i}^{2}}\right)^{1 / 3} X_{i}^{2+5 \lambda_{i}}
$$

The delay of intersection is formulated as

$$
d_{\text {intersection }}=\frac{\sum d_{i} q_{i}}{\sum q_{i}}
$$

3.2. Environmental Effect. The optimization objective of environmental effect aims at vehicle emission (VE) and energy consumption (EC). Due to the worse global environment, it should pay more attention to the environmental.

(a) Vehicle Emission. The main goal of vehicle emission based optimization is to reduce environmental pollution. Some standard assumptions are made as in most vehicle emission calculation models [21, 33-35]:

(i) the arrival rate of cars, arriving at the intersection, is stable; the diverging rate of cars, driving away from the intersection, is stable; the cars, waiting for green light, park in equal interval queues;

(ii) the main state of cars, passing through the intersection, only includes uniform motion state, uniform variable motion, and idling;

(iii) the basic process of cars passing intersection includes deceleration process (Phase $a$ ), idling process (Phase $b$ ), acceleration process (Phase $c$ ), and uniform speed process (Phase $d$ );

(iv) this paper will not enter the effect of pollutant diffusion for the moment.

In Phase $a$, travel time and travel distance of cars driving in group $i$ are easy to calculate:

$$
\begin{gathered}
t_{i, a}=\frac{v_{i}}{a_{\text {deceleration }}}, \\
D_{i, a}=\frac{v_{i} t_{i, a}}{2} .
\end{gathered}
$$

The whole deceleration time for group $i$ in Phase $a$ needs to be revised by parking rate; namely,

$$
T_{i, a}=t_{i, a} q_{i} h_{i}
$$

The parking rate for lane group $i$ is stated as shown [32]:

$$
h_{i}=\frac{1-\lambda_{i}}{1-X_{i}}
$$

The basic characteristics of Phase $c$ are the same as that of Phase $a$. Thus,

$$
\begin{gathered}
t_{i, c}=\frac{v_{i}}{a_{\text {acceleration }}}, \\
D_{i, c}=\frac{v_{i} t_{i, c}}{2}, \\
T_{i, c}=t_{i, c} q_{i} h_{i} .
\end{gathered}
$$

In Phase $d$, travel distance and travel time of cars driving in group $i$ are easy to calculate:

$$
\begin{gathered}
D_{i, d}=L_{i}-D_{i, a}-D_{i, b}-D_{i, c}, \\
t_{i, d}=\frac{D_{i, d}}{v_{i}} .
\end{gathered}
$$

The whole deceleration time for group $i$ in Phase $d$ needs to be revised by parking rate; namely,

$$
\begin{aligned}
T_{i, d} & =\frac{q_{i}\left(1-h_{i}\right) L_{i}}{v_{i}}+\frac{q_{i} h_{i} D_{i, d}}{v_{i}} \\
& =\frac{q_{i} L_{i}-q_{i} h_{i}\left(D_{i, a}+D_{i, b}+D_{i, c}\right)}{v_{i}} .
\end{aligned}
$$

In Phase $b$, travel distance and travel time of cars driving in group $i$ are easy to calculate:

$$
\begin{gathered}
D_{i, b}=0, \\
t_{i, b}=\frac{d_{i}}{h_{i}}-\left(t_{i, a}+t_{i, c}-\frac{D_{i, a}+D_{i, b}+D_{i, c}}{v_{i}}\right)=\frac{d_{i}}{h_{i}}-\frac{t_{i, a}+t_{i, c}}{2} .
\end{gathered}
$$

The whole deceleration time for group $i$ in Phase $b$ needs to be revised by parking rate; namely,

$$
T_{i, b}=q_{i} d_{i}-q_{i} h_{i} \frac{t_{i, a}+t_{i, c}}{2} .
$$

The emission quality of gas $k$ for group $i$ is stated as shown [34]:

$$
E_{i, k}=\mathrm{EF}_{k, i, a} \cdot T_{i, a}+\mathrm{EF}_{k, i, b} \cdot T_{i, b}+\mathrm{EF}_{k, i, c} \cdot T_{i, c}+\mathrm{EF}_{k, i, d} \cdot T_{i, d} .
$$

Therefore, the emission quality of gas $k$ is formulated as

$$
E_{k}=\sum E_{i, k}
$$

Then, emission equivalent of intersection is formulated as

$$
E_{\text {intersection }}=\sum \alpha_{k} E_{k} \text {. }
$$

(b) Energy Consumption. The main goal of energy consumption based optimization is to reduce resource waste. Basic assumptions of energy consumption are the same as that of vehicle emission. [36]:

The energy consumption for group $i$ is stated as shown

$$
C_{i}=\mathrm{CF}_{i, a} \cdot T_{i, a}+\mathrm{CF}_{i, b} \cdot T_{i, b}+\mathrm{CF}_{i, c} \cdot T_{i, c}+\mathrm{CF}_{i, d} \cdot T_{i, d} .
$$


Then, energy consumption of intersection is formulated as

$$
C_{\text {intersection }}=\sum C_{i}
$$

3.3. Restrictive Conditions. Restrictive conditions consist of the following aspects.

(a) Physical Space. Considering high cost of rebuilding, limitations of terrain condition, and the other elements, there are physical space restrictions. The number of lanes satisfies the following conditions:

$$
\begin{aligned}
& m_{1}=n_{1}+n_{6}+n_{9}+n_{13}, \\
& m_{2}=n_{3}+n_{8}+n_{10}+n_{14}, \\
& m_{3}=n_{2}+n_{5}+n_{11}+n_{15}, \\
& m_{4}=n_{4}+n_{7}+n_{12}+n_{16}, \\
& n_{13}=n_{2}+n_{10}, \\
& n_{14}=n_{4}+n_{11}, \\
& n_{15}=n_{6}+n_{12}, \\
& n_{16}=n_{8}+n_{9}, \\
& m_{1} \leq M_{1}, \\
& m_{2} \leq M_{2}, \\
& m_{3} \leq M_{3}, \\
& m_{4} \leq M_{4}, \\
& n_{i}, m_{j}, M_{j} \in N^{*} .
\end{aligned}
$$

(b) Signal Control. Considering signal switching rules, signal control machine parameters, traffic safety, and the other elements, there are signal control restrictions.

For traditional four-phase setting (overlapping), the green time of group $i$ satisfies the following conditions:

$$
\begin{gathered}
t g_{1}+t g_{2}=t g_{5}+t g_{6}, \\
t g_{3}+t g_{4}=t g_{7}+t g_{8}, \\
t g_{1}+t g_{2}+t g_{3}+t g_{4}=C-4 t_{\text {all-red }}-4 t_{\text {yellow }} \\
t_{\text {all-red }}=2, \quad t_{\text {yellow }}=4
\end{gathered}
$$

For signal control machine, cycle length is normally limited within a certain range. In the proposed optimization model, cycle length restriction is reflected in constraint conditions, as shown:

$$
C_{\min } \leq C \leq C_{\max }
$$

The typical value of minimum green time would be 4 seconds [37]. However, if pedestrian timing requirements exist, the minimum green time for a phase is estimated by $G_{P}$
[31]. Thus, the green time of group $i$ also satisfies the following condition:

$$
\operatorname{tg}_{i} \geq \max \left[G_{P}, 4\right]
$$

(c) Maximum Saturation. Maximum saturation is used to ensure high capacity. Consider

$$
X_{i} \leq X_{\max }
$$

(d) Maximum Delay. Maximum delay is used to ensure high traffic feeling. Consider

$$
d_{i} \leq d_{\max }
$$

\section{Automation Countermeasure System Design}

4.1. System Operation Interfaces. Based on system organization and methodology, system operation interfaces include the following.

(a) Lane Settings. It is used to input the static data.

(b) Volume and Timing Settings. It is used to input dynamic data.

(c) Evaluation Settings. It is used to choose the structure of the MOP model.

(d) Comparative Analysis. Some evaluation data, achieved by investigation, are input into system in this operation interface. Moreover, it provides comparative analysis of optimization schemes (OS) and present scheme (PS).

\subsection{Structure of the MOP Model}

4.2.1. Single Objective Optimization. Considering the users may have different attention degree for the proposed optimization objectives, ACSIO provides single objective optimization, namely, the following.

(a) Capacity Based Optimization. The capacity based optimization is formulated as

$$
\begin{array}{ll}
\max & \mathrm{ME}=\sum s_{i} \frac{g_{i}}{C} \\
\text { s.t. } & \sum\left(\bar{X}_{i}-X_{i}\right)^{2} \leq X_{0} \\
& X_{i} \leq X_{\max }, \\
& d_{i} \leq d_{\max }, \\
& n_{1}+n_{6}+n_{9}+n_{2}+n_{10} \leq M_{1}, \\
& n_{3}+n_{8}+n_{10}+n_{4}+n_{11} \leq M_{2}, \\
& n_{2}+n_{5}+n_{11}+n_{6}+n_{12} \leq M_{3}, \\
& n_{4}+n_{7}+n_{12}+n_{8}+n_{9} \leq M_{4},
\end{array}
$$




$$
\begin{aligned}
& \operatorname{tg}_{1}+t_{g_{2}}-t g_{5}-t g_{6}=0, \\
& \operatorname{tg}_{3}+\operatorname{tg}_{4}-t g_{7}-t g_{8}=0, \\
& \operatorname{tg}_{1}+t g_{2}+t g_{3}+\operatorname{tg}_{4}+24 \leq C_{\max }, \\
& \operatorname{tg}_{1}+\operatorname{tg}_{2}+\operatorname{tg}_{3}+\operatorname{tg}_{4}+24 \geq C_{\min }, \\
& \operatorname{tg}_{i} \geq \max \left[G_{P}, 4\right], \\
& \operatorname{tg}_{i} \in N^{*}, \\
& n_{i} \in N^{*} .
\end{aligned}
$$

(b) Delay Based Optimization. The delay based optimization is formulated as

$$
\begin{array}{ll}
\min & \text { TF }=\frac{\sum d_{i} q_{i}}{\sum q_{i}}, \\
\text { s.t. } & X_{i} \leq X_{\max }, \\
& d_{i} \leq d_{\max }, \\
& n_{1}+n_{6}+n_{9}+n_{2}+n_{10} \leq M_{1}, \\
& n_{3}+n_{8}+n_{10}+n_{4}+n_{11} \leq M_{2}, \\
& n_{2}+n_{5}+n_{11}+n_{6}+n_{12} \leq M_{3}, \\
& n_{4}+n_{7}+n_{12}+n_{8}+n_{9} \leq M_{4}, \\
& \operatorname{tg}_{1}+\operatorname{tg}_{2}-\operatorname{tg}_{5}-\operatorname{tg}_{6}=0, \\
& \operatorname{tg}_{3}+\operatorname{tg}_{4}-\operatorname{tg}_{7}-\operatorname{tg}_{8}=0, \\
& \operatorname{tg}_{1}+\operatorname{tg}_{2}+\operatorname{tg}_{3}+\operatorname{tg}_{4}+24 \leq C_{\max }, \\
& \operatorname{tg}_{1}+\operatorname{tg}_{2}+\operatorname{tg}_{3}+\operatorname{tg}_{4}+24 \geq C_{\min }, \\
& \operatorname{tg}_{i} \geq \max \left[G_{P}, 4\right], \\
& \operatorname{tg}_{i} \in N^{*}, \\
& n_{i} \in N^{*} .
\end{array}
$$

(c) Vehicle Emission Based Optimization. The vehicle emission based optimization is formulated as

$$
\min \mathrm{VE}=\sum \alpha_{k} E_{k}
$$

The restrictive conditions of formulation (28) are the same as that of formulation (27).

(d) Energy Consumption Based Optimization. The energy consumption based optimization is formulated as

$$
\min \mathrm{EC}=\sum C_{i}
$$

The restrictive conditions of formulation (29) are the same as that of formulation (27).

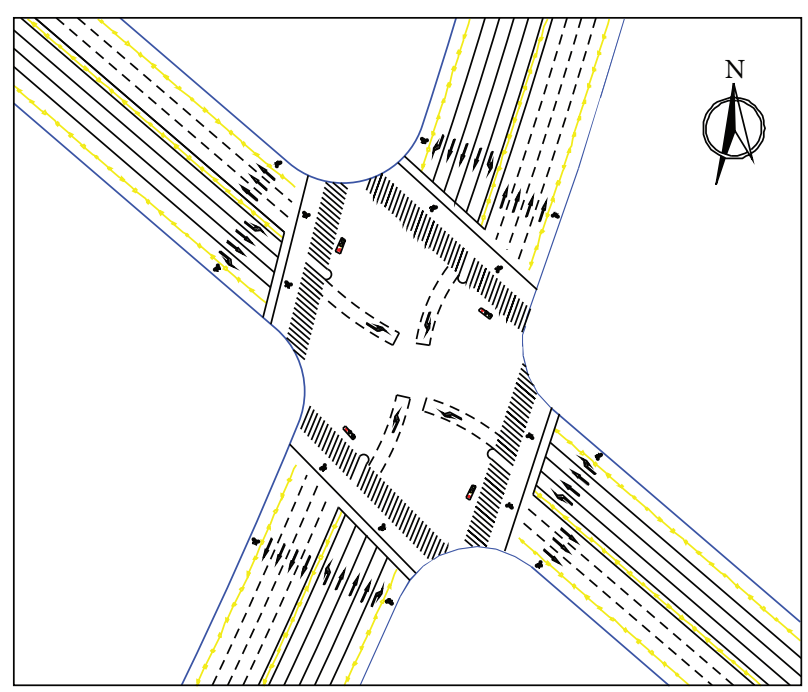

FIGURE 3: Research object.

4.2.2. Multiobjective Optimization. ACSIO also provides multiobjective optimization with adjustable weight, as shown:

$$
\max \left(\varphi_{\mathrm{ME}} \mathrm{ME}+\varphi_{\mathrm{TF}} \frac{1}{\mathrm{TF}}+\varphi_{\mathrm{VE}} \frac{1}{\mathrm{VE}}+\varphi_{\mathrm{EC}} \frac{1}{\mathrm{EC}}\right)
$$

The restrictive conditions of formulation (30) are the same as that of formulation (26). The intersection optimization is turned into single objective optimization problem. $\varphi_{\mathrm{ME}}, \varphi_{\mathrm{TF}}, \varphi_{\mathrm{VE}}$, and $\varphi_{\mathrm{EC}}$ are assigned by users' knowledge.

\section{Case Study}

5.1. Data Characteristics. Taking an intersection as research object, it verifies the effectiveness and feasibility of the proposed method. Basic data is achieved by investigation. The static data of the intersection is shown in Figure 3.

5.2. Computational Process. Take capacity based optimization as an example to explain the computational process. Present scheme is designed based on HCM 2000. Optimization scheme is designed based on ACSIO.

(a) Optimization for Morning Peak. As shown in Table 3, the lane and green time are changed in optimization scheme.

(b) Optimization for Common. As shown in Table 4, the lane and green time are changed in optimization scheme.

(c) Optimization for Evening Peak. As shown in Table 5, the lane and green time are changed in optimization scheme.

5.3. Results. Comparative analysis of present scheme and optimization scheme is carried out from two aspects, namely, scheme design and evaluation parameters. Taking saturation 
TABLE 3: Optimization for morning peak.

\begin{tabular}{|c|c|c|c|c|c|}
\hline \multicolumn{2}{|c|}{ Approach } & \multicolumn{2}{|c|}{ Lane } & \multicolumn{2}{|c|}{ Green time (s) } \\
\hline & & PS & OS & PS & OS \\
\hline \multirow{4}{*}{ North } & Through & 3 & 3 & 43 & 31 \\
\hline & Right & 1 & 1 & 150 & 110 \\
\hline & Left & 1 & 2 & 21 & 11 \\
\hline & Export & 4 & 3 & & \\
\hline \multirow{4}{*}{ South } & Through & 3 & 2 & 43 & 28 \\
\hline & Right & 1 & 1 & 150 & 110 \\
\hline & Left & 1 & 2 & 21 & 8 \\
\hline & Export & 4 & 4 & & \\
\hline \multirow{4}{*}{ East } & Through & 2 & 2 & 41 & 34 \\
\hline & Right & 1 & 1 & 150 & 110 \\
\hline & Left & 1 & 1 & 21 & 24 \\
\hline & Export & 3 & 3 & & \\
\hline \multirow{4}{*}{ West } & Through & 2 & 2 & 41 & 23 \\
\hline & Right & 1 & 1 & 150 & 110 \\
\hline & Left & 1 & 1 & 21 & 13 \\
\hline & Export & 3 & 3 & & \\
\hline
\end{tabular}

TABLE 4: Optimization for common.

\begin{tabular}{|c|c|c|c|c|c|}
\hline \multicolumn{2}{|c|}{ Approach } & \multicolumn{2}{|c|}{ Lane } & \multicolumn{2}{|c|}{ Green time (s) } \\
\hline & & PS & OS & PS & OS \\
\hline \multirow{4}{*}{ North } & Through & 3 & 3 & 43 & 31 \\
\hline & Right & 1 & 1 & 150 & 114 \\
\hline & Left & 1 & 2 & 21 & 10 \\
\hline & Export & 4 & 3 & \multicolumn{2}{|c|}{-} \\
\hline \multirow{4}{*}{ South } & Through & 3 & 2 & 43 & 33 \\
\hline & Right & 1 & 2 & 150 & 114 \\
\hline & Left & 1 & 1 & 21 & 12 \\
\hline & Export & 4 & 4 & \multicolumn{2}{|c|}{ - } \\
\hline \multirow{4}{*}{ East } & Through & 2 & 2 & 41 & 26 \\
\hline & Right & 1 & 1 & 150 & 114 \\
\hline & Left & 1 & 1 & 21 & 17 \\
\hline & Export & 3 & 3 & \multicolumn{2}{|c|}{ - } \\
\hline \multirow{4}{*}{ West } & Through & 2 & 2 & 41 & 30 \\
\hline & Right & 1 & 1 & 150 & 114 \\
\hline & Left & 1 & 1 & 21 & 21 \\
\hline & Export & 3 & 3 & \multicolumn{2}{|c|}{-} \\
\hline
\end{tabular}

and delay as evaluation parameters, the results of optimization are obtained as shown in Figure 4.

(a) Synthesis Optimization for Channelization and Signal Control. Parameters of channelization and signal control are all output data of ACSIO. As shown in Tables 3-5, the lane and green time are changed. Average value of saturation and delay for different lane groups shows that the optimization scheme has a higher coordination between channelization and signal control.

(b) Dynamic Management for Dynamic Traffic Demand. Traffic demands of morning peak, common, and evening peak are different. As shown in Figure 4, fixed channelization plan is no lager suitable for dynamic traffic management. Lane use control for tide traffic, which is shown in
TABLE 5: Optimization for evening peak.

\begin{tabular}{lccccc}
\hline \multicolumn{2}{c}{ Approach } & \multicolumn{2}{c}{ Lane } & \multicolumn{2}{c}{ Green time (s) } \\
& & PS & OS & PS & OS \\
\hline \multirow{4}{*}{ North } & Through & 3 & $\mathbf{2}$ & 43 & $\mathbf{2 6}$ \\
& Right & 1 & 1 & 150 & $\mathbf{1 1 5}$ \\
& Left & 1 & 1 & 21 & $\mathbf{1 6}$ \\
& Export & 4 & $\mathbf{5}$ & & - \\
\hline \multirow{4}{*}{ South } & Through & 3 & $\mathbf{4}$ & 43 & $\mathbf{2 8}$ \\
& Right & 1 & 1 & 150 & $\mathbf{1 1 5}$ \\
& Left & 1 & 1 & 21 & $\mathbf{1 8}$ \\
& Export & 4 & $\mathbf{3}$ & & - \\
\hline \multirow{4}{*}{ East } & Through & 2 & 2 & 41 & $\mathbf{2 8}$ \\
& Right & 1 & 1 & 150 & $\mathbf{1 1 5}$ \\
& Left & 1 & 1 & 21 & $\mathbf{2 2}$ \\
\multirow{4}{*}{ West } & Export & 3 & 3 & & - \\
& Through & 2 & 2 & 41 & $\mathbf{2 5}$ \\
& Right & 1 & 1 & 150 & $\mathbf{1 1 5}$ \\
& Left & 1 & 1 & 21 & $\mathbf{1 9}$ \\
& Export & 3 & 2 & \multicolumn{2}{c}{-} \\
\hline
\end{tabular}

Tables 3-5, has a higher coordination between dynamic management and dynamic traffic demand.

(c) Pertinence Optimization for Traffic Problems. As shown in Figure 4, oversaturated lane group exists in present scheme. Traffic efficiency, especially the managerial effectiveness, is the main problem of present scheme. Pertinence optimization is done based on ACSIO. As shown in Figure 4, optimization scheme has a higher coordination between scheme design and traffic problems.

(d) Adjustable MOP Model Solution. It embodies the operability of ACSIO. This paper only takes capacity based optimization as an example, because the computational processes of different optimization theories shown in Section 5 are basically identical. In the future studies, more experiments will be done to test the efficiency and applicability of the proposed modeling and computing methods.

\section{Conclusions}

The main contribution of this paper is to establishing an automation countermeasure system for intersection optimization (ACSIO). Automation countermeasure system organization is proposed based on intersection optimization problem statement. Moreover, methodology is put forward according to system function and architecture. Then, automation countermeasure system design is given. The core of ACSIO is listed as follows:
(a) decision support for intersection management;
(b) synthesis optimization for channelization and signal control;
(c) dynamic management for dynamic traffic demand;
(d) pertinence optimization for traffic problems;
(e) adjustable MOP model solution. 

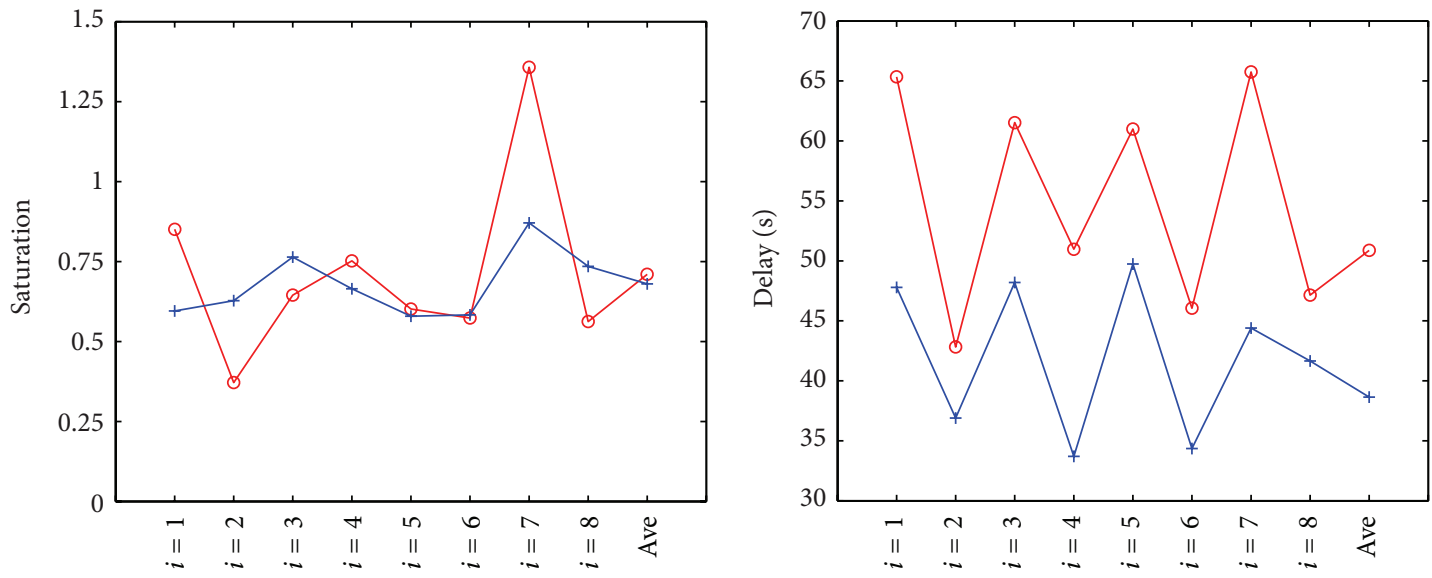

(a) Optimization for morning peak
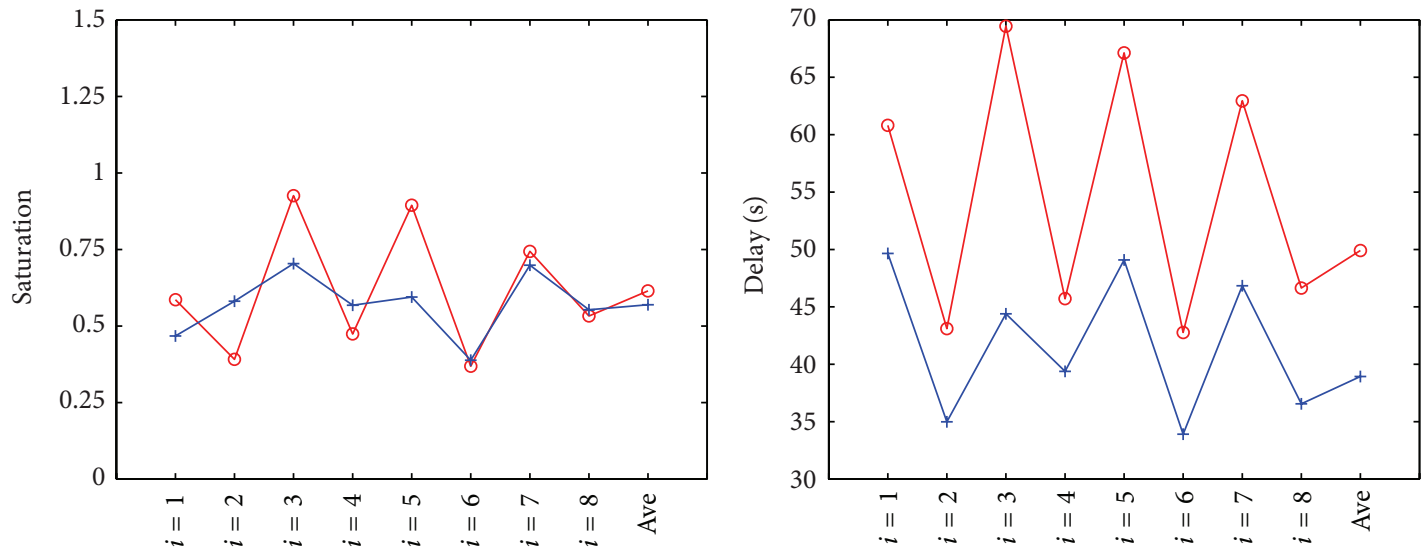

(b) Optimization for common
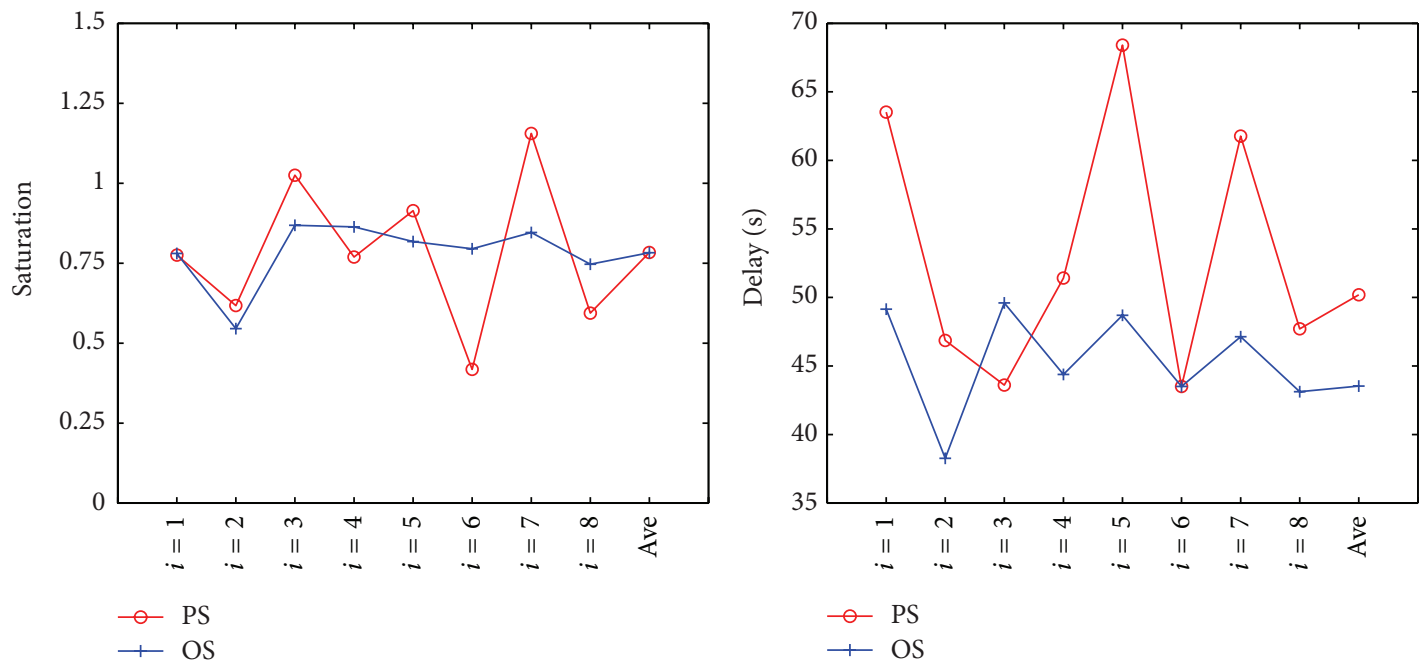

(c) Optimization for evening peak

FIGURE 4: Optimization results. 
Several extensions may be considered. Mathematic model needs to be built to describe the relationship between these different optimization objectives. Besides, microscopic simulation model needs to be established to evaluate the optimization scheme more persuasively.

\section{Notations}

$i$ :

$j:$

$m_{j}$ :

$n_{i}:$

$c_{i}$ :

$s_{i}:$

$g_{i}:$

$C:$

$\lambda_{i}:$

$c_{\text {intersection }}$ :

$X_{i}:$

$q_{i}:$

$\bar{X}_{i}$ :

$X_{0}$ :

$d_{i}$ :

$d_{\text {intersection }}$ :

$t_{i, a}, t_{i, b}, t_{i, c}$, and $t_{i, d}$ :

$v_{i}$ :

$a_{\text {deceleration: }}$

$a_{\text {acceleration }}:$

$L_{i}$ :

$D_{i, a}, D_{i, b}, D_{i, c}$, and $D_{i, d}$ :

$h_{i}:$

$T_{i, a}, T_{i, b}, T_{i, c}$, and $T_{i, d}$ :

$\mathrm{EF}_{k, i, a}, \mathrm{EF}_{k, i, b}, \mathrm{EF}_{k, i, c}$, and $\mathrm{EF}_{k, i, d}$ :
Group number

Approach number

Number of lanes for

approach $j$

Number of lanes for

group $i$

Capacity of lane group $i$ (veh/h)

Saturation flow rate for

lane group $i$ (veh/h)

Effective green time for

lane group $i$ (s)

Cycle length (s)

Green ratio for lane group

$i$

Capacity of intersection

(veh/h)

Saturation for lane group $i$

Actual or projected

demand flow rate for lane

group $i$ (veh/h)

Average value of $X_{i}$

$D$-value threshold of

variance of $X_{i}$

Delay for lane group $i$ (s)

Delay of intersection (s)

Travel time of cars driving in group $i$ in Phases $a, b$, $c$, and $d(\mathrm{~s})$

Travel speed of cars passing intersection in uniform speed $(\mathrm{m} / \mathrm{s})$

Deceleration of cars

driving in Phase $a\left(\mathrm{~m} / \mathrm{s}^{2}\right)$

Acceleration of cars

driving in Phase $c\left(\mathrm{~m} / \mathrm{s}^{2}\right)$

Travel distance of cars

passing intersection in

group $i(\mathrm{~m})$

Travel distance of cars

driving in group $i$ in

Phases $a, b, c$, and $d(\mathrm{~m})$

Parking rate for lane

group $i$

Whole deceleration time for group $i$ in Phases $a, b$, $c$, and $d(\mathrm{~s})$

Emission factor of gas $k$

for group $i$ in Phases $a, b$, $c$, and $d(\mathrm{mg} / \mathrm{s})$

\begin{tabular}{|c|c|}
\hline$E_{i, k}:$ & $\begin{array}{l}\text { Emission quality of gas } k \text { for } \\
\text { group } i(\mathrm{~kg})\end{array}$ \\
\hline$E_{k}:$ & Emission quality of gas $k(\mathrm{~kg})$ \\
\hline$\alpha_{k}:$ & $\begin{array}{l}\text { Emission equivalent value of } \\
\text { gas } k\end{array}$ \\
\hline$E_{\text {intersection }}:$ & $\begin{array}{l}\text { Emission equivalent of inter- } \\
\text { section }(\mathrm{kg})\end{array}$ \\
\hline $\mathrm{CF}_{i, a}, \mathrm{CF}_{i, b}, \mathrm{CF}_{i, c}$, and $\mathrm{CF}_{i, d}$ : & $\begin{array}{l}\text { Energy consumption factor for } \\
\text { group } i \text { in Phases } a, b, c \text {, and } d \\
\text { (gal/s) }\end{array}$ \\
\hline$C_{i}:$ & $\begin{array}{l}\text { Energy consumption quality } \\
\text { for group } i \text { (gal) }\end{array}$ \\
\hline$C_{\text {intersection }}:$ & $\begin{array}{l}\text { Energy consumption quality } \\
\text { of intersection (gal) }\end{array}$ \\
\hline$M_{j}:$ & $\begin{array}{l}\text { Restricted number of lanes for } \\
\text { approach } j\end{array}$ \\
\hline $\operatorname{tg}_{i}:$ & Green time for lane group $i(\mathrm{~s})$ \\
\hline$t_{\text {all-red }}:$ & All-red time (s) \\
\hline$t_{\text {yellow }}:$ & Yellow time (s) \\
\hline$C_{\min }:$ & $\begin{array}{l}\text { Lower limit value of cycle } \\
\text { length }(s)\end{array}$ \\
\hline$C_{\max }:$ & $\begin{array}{l}\text { Upper limit value of cycle } \\
\text { length (s) }\end{array}$ \\
\hline$G_{P}:$ & $\begin{array}{l}\text { Minimum green time for pe- } \\
\text { destrian crossing (s) }\end{array}$ \\
\hline$X_{\max }:$ & Maximum saturation \\
\hline$d_{\max }:$ & Maximum delay (s) \\
\hline$\varphi_{\mathrm{ME}}, \varphi_{\mathrm{TF}}, \varphi_{\mathrm{VE}}$, and $\varphi_{\mathrm{EC}}:$ & Adjustable weight. \\
\hline
\end{tabular}

\section{Conflict of Interests}

The authors declare that there is no conflict of interests regarding the publication of this paper.

\section{Acknowledgments}

This research was funded by the National Natural Science Foundation of China (no. 51408023) and Beijing Science and Technology Plan (no. Z121100000312101).

\section{References}

[1] Beijing Yearbook Publishing House, Beijing Yearbook 2013, Beijing Yearbook Publishing House, Beijing, China, 2013.

[2] Beijing Transportation Research Center, "Beijing road traffic operation analysis report (2014.5)," Tech. Rep., Beijing Transportation Research Center, Beijing, China, 2014.

[3] H. P. Lu, "Urban traffic congestion mechanism analysis and counter measure system," Comprehensive Transportation, no. 3, pp. 10-19, 2014.

[4] Z. Y. Sun, Research on Urban Intersection Signal Control Methods Based on Engineering Practice in Beijing, Beijing Jiaotong University, Beijing, China, 2012.

[5] L. Fan, "Measures of channelization design of urban road grade intersection," Urban Roads Bridges \& Flood Control, vol. 184, no. 8, pp. 6-29, 2014.

[6] L. Du and Y. Zhang, "Positive switched system approach to traffic signal control for oversaturated intersection," Discrete 
Dynamics in Nature and Society, vol. 2014, Article ID 381567, 8 pages, 2014.

[7] Y. J. Feng, M. Shan, H. C. Le, G. J. Zhang, and L. Yu, "Subarea dynamic division algorithm based on green wave coordinated control," Control Theory \& Applications, vol. 31, no. 5, pp. 10341046, 2014.

[8] Y. R. Bi, D. Srinivasan, X. B. Lu, Z. Sun, and W. L. Zeng, “Type2 fuzzy multi-intersection traffic signal control with differential evolution optimization," Expert Systems with Applications, vol. 41, no. 16, pp. 7338-7349, 2014.

[9] C. Yan, H. Jiang, and S. Xie, "Capacity optimization of an isolated intersection under the phase swap sorting strategy," Transportation Research Part B: Methodological, vol. 60, pp. 85106, 2014.

[10] J. F. Dai, G. H. Zhang, N. Zhai, and H. Li, "Improvement and practice in urban traffic engineering design," Urban Transport of China, vol. 9, no. 1, pp. 40-46, 2011.

[11] X. Zeng, Y. Zhang, K. N. Balke, and K. Yin, "A real-time transit signal priority control model considering stochastic bus arrival time," IEEE Intelligent Transportation Systems Magazine, vol. 15, no. 4, pp. 1657-1666, 2014.

[12] X. Zhang and G. L. Chang, "A mixed-flow simulation model for congested intersections with high pedestrian-vehicle traffic flows," Simulation: Transactions of the Society for Modeling and Simulation International, vol. 90, no. 5, pp. 570-590, 2014.

[13] X. Bing, Y. Jiang, C. Zhang, Y. Zhang, and J. Lu, "Effects of intersection lane configuration on traffic emissions," Advances in Transportation Studies, no. 32, pp. 23-36, 2014.

[14] K. Xie and X. S. Wang, "Signalized intersection safety analysis using Bayesian hierarchical model," China Journal of Highway and Transport, vol. 27, no. 2, pp. 90-97, 2014.

[15] X. N. Zhu and B. Long, "Bi-level programming model of timing optimization for multiple bus priority intersection," Journal of Traffic and Transportation Engineering, vol. 14, no. 1, pp. 103-111, 2014.

[16] Q. Long, L. G. Hu, J. F. Zhang, Z. M. Zhou, and S. Peng, "Multiobjective optimization based on traffic management strategy for intersection signal controlling," Journal of Central South University (Science and Technology), vol. 45, no. 7, pp. 25032508, 2014.

[17] A. M. Roshandeh, H. S. Levinson, Z. Z. Li, H. Patel, and B. Zhou, "New methodology for intersection signal timing optimization to simultaneously minimize vehicle and pedestrian delays," Journal of Transportation Engineering, vol. 140, no. 5, Article ID 04014009, 2014.

[18] Y. F. Gao, H. Hu, H. Han, and X. G. Yang, "Multi-objective optimization and simulation for urban road intersection group traffic signal control," China Journal of Highway and Transport, vol. 25, no. 6, pp. 129-135, 2012.

[19] J. G. Hao, Z. S. Hou, and X. B. Liu, "Vehicle queuing length balanced-control of the signalized isolated intersection via multi-parametric programming," Control and Decision, vol. 28, no. 4, pp. 595-599, 2013.

[20] H. S. Qi, D. H. Wang, and S. Chen, "Isolated intersection control based on integrated saturation degree," Journal of Harbin Institute of Technology, vol. 44, no. 2, pp. 134-137, 2012.

[21] Q. X. Cao, W. Yang, and Z. W. Wang, "Intersection signal automation optimization considering vehicle emission," System Engineering, vol. 31, no. 9, pp. 68-72, 2013.

[22] Y. H. Liu, H. B. Liao, Z. Yu, and M. Cai, "Study of intersection control mode evaluation based on environmental effect," Acta
Scientiarum Natralium Universitatis Sunyatseni, vol. 52, no. 1, pp. 12-16, 2013.

[23] J. J. Shen and W. Wang, "Study on the complexity degree model based on the dynamic characteristics of traffic flow," China Civil Engineering Journal, vol. 44, no. 5, pp. 131-135, 2011.

[24] H. P. Lu, The Automation of Traffic Countermeasure System, Tsinghua University, Beijing, China, 2014.

[25] H. P. Lu, Feasibility Survey about the Automated Solution of the Traffic Bottleneck, Tsinghua University, Beijing, China, 2014.

[26] Beijing Transportation Research Center, "2013 Beijing transport annual report," Tech. Rep., Beijing Transportation Research Center, Beijing, China, 2013.

[27] Ministry of Environmental Protection, 2013 China Environment Condition Communique, Ministry of Environmental Protection, Beijing, China, 2014.

[28] Traffic Management Bureau of the Ministry of Public Security, "2013 China road traffic accident statistics annual report," Tech. Rep., Traffic Management Bureau of the Ministry of Public Security, Beijing, China, 2014.

[29] R. M. Li, H. P. Lu, and M. Guo, "Urban road basic geographic elements coding methodology research," Central South Highway Engineering, vol. 34, no. 5, pp. 37-40, 2009.

[30] H. J. Li, H. H. Dong, P. F. Zhang, L. M. Jia, Y. G. Zhang, and Y. Qin, "Semantic coding of sensor network nodes for road traffic," Journal of Central South University (Science and Technology), vol. 44, no. 6, pp. 2368-2377, 2013.

[31] Transportation Research Board, 2000 Highway Capacity Manual, Transportation Research Board of the National Academies, Washington, DC, USA, 2000.

[32] F. V. Webster, Traffic Signal Settings, Road Laboratory Technical Paper, no. 39, 1958.

[33] W. T. Zhao, W. Yang, and Y. B. Yue, "Optimization research on signal timing for urban intersection by considering vehicle emission," Journal of Wuhan University of Technology (Transportation Science \& Engineering), vol. 36, no. 5, pp. 911-915, 2012.

[34] H. B. Gong, Multi-Objective Optimization Model of Intersection Signal Control Considering Vehicle Emission Characteristics, Beijing Jiaotong University, Beijing, China, 2014.

[35] J. H. Shi, Analysis and Comprehensive Evaluation on Traffic Pollution at Urban Road Intersection, Jilin University, Changchun, China, 2012.

[36] W. H. Zhang, Q. Q. Li, and S. K. Zhang, "Optimized timing method of signal intersection based on cumulative energy consumption," Journal of East China Jiaotong University, vol. 31, no. 3, pp. 42-49, 2014.

[37] Trafficware, Synchro Studio 8 User Guide, Trafficware, Sugar Land, Tex, USA, 2011. 


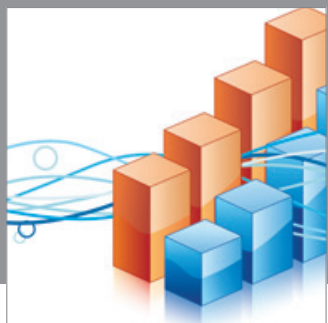

Advances in

Operations Research

mansans

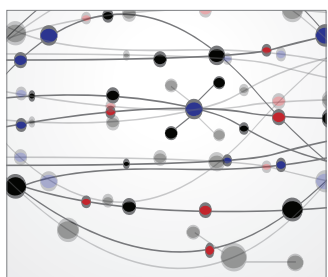

The Scientific World Journal
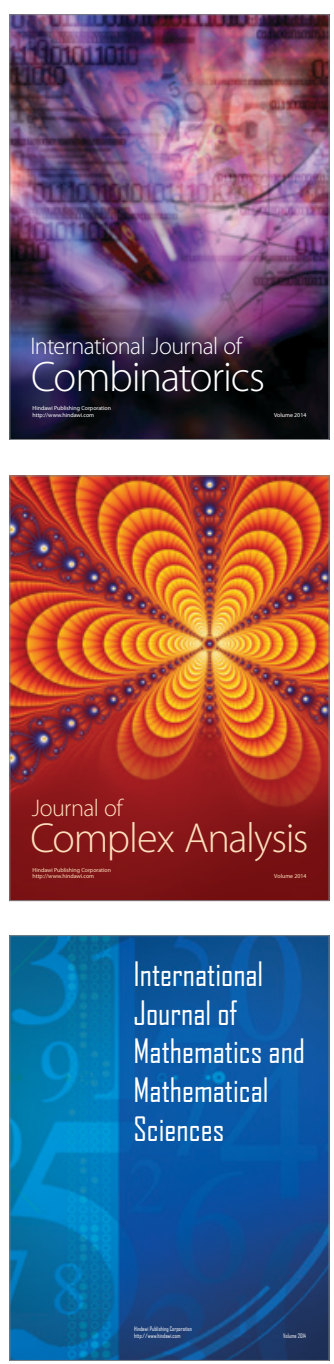
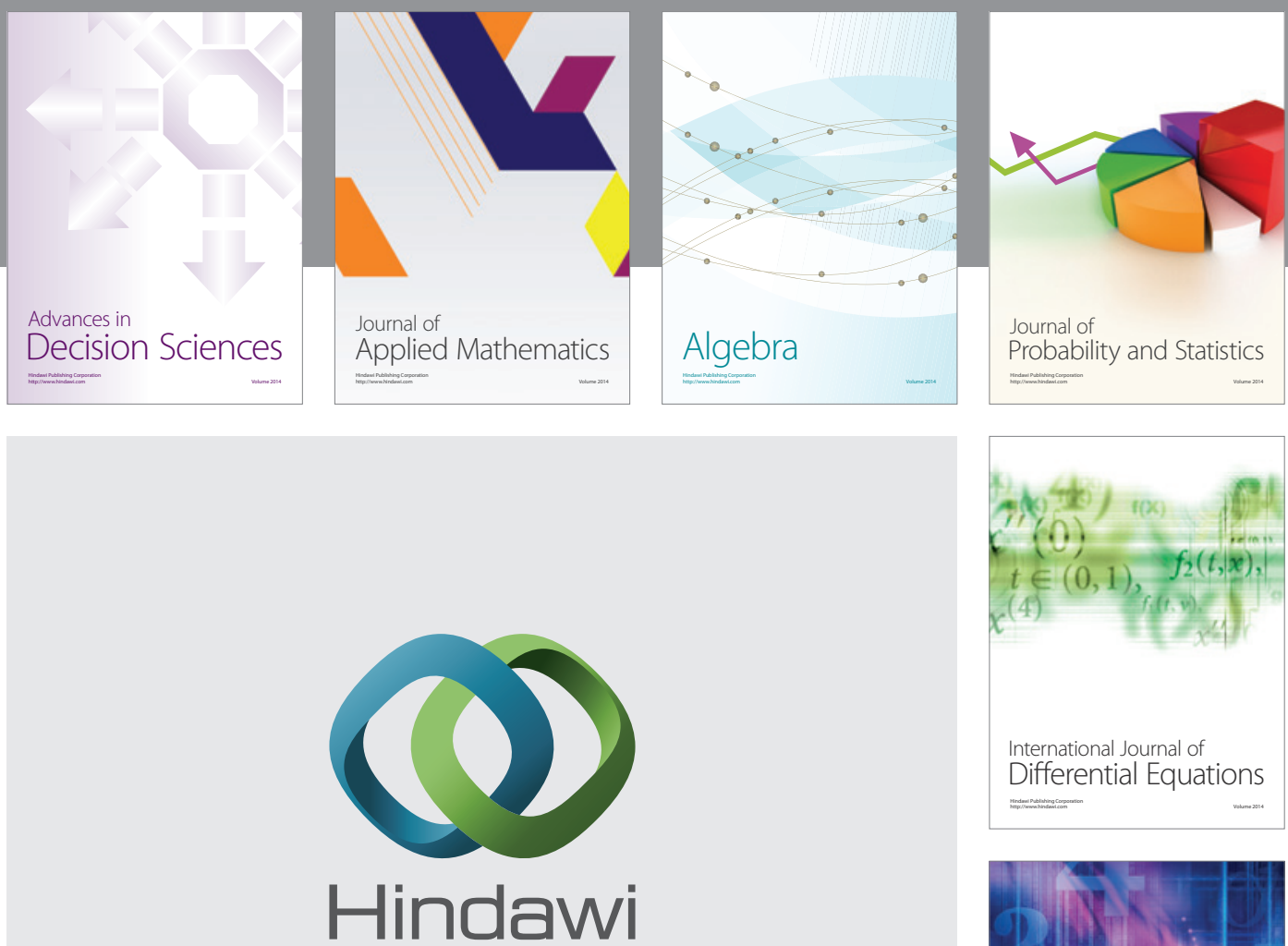

Submit your manuscripts at http://www.hindawi.com
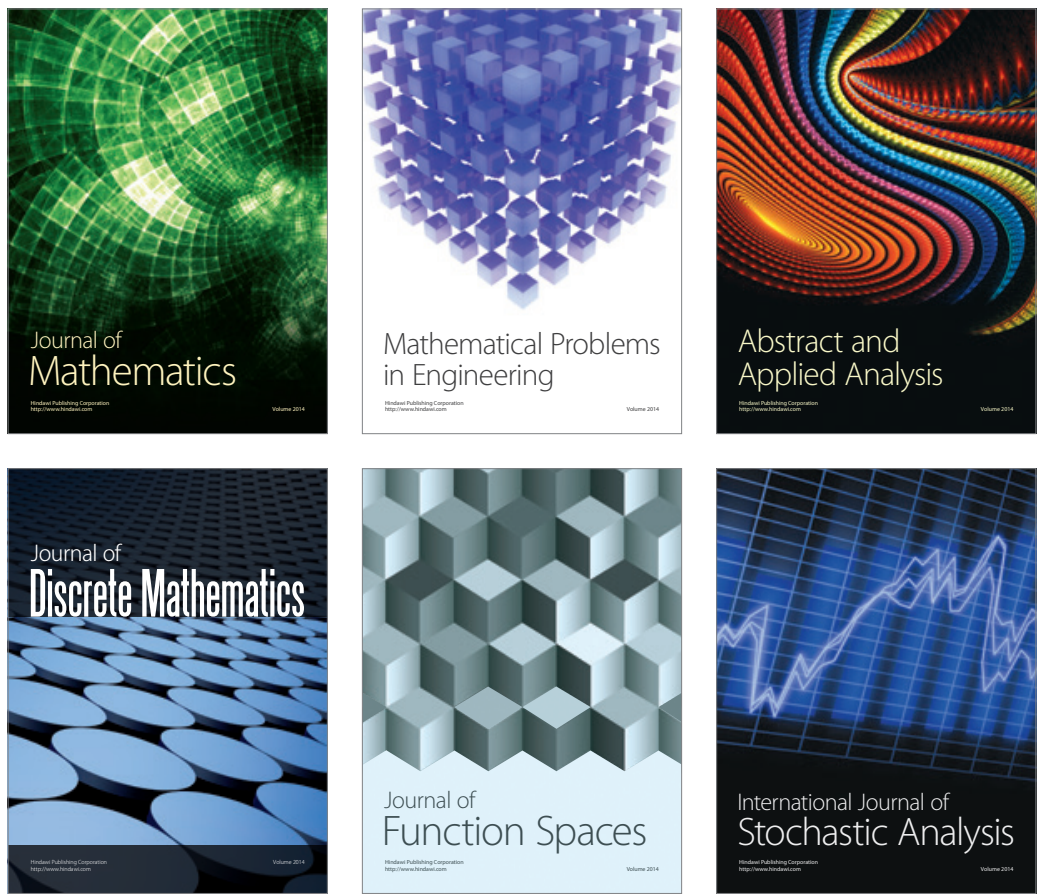

Journal of

Function Spaces

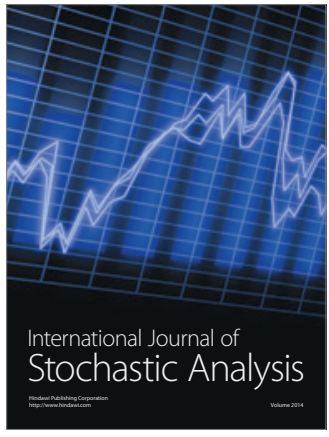

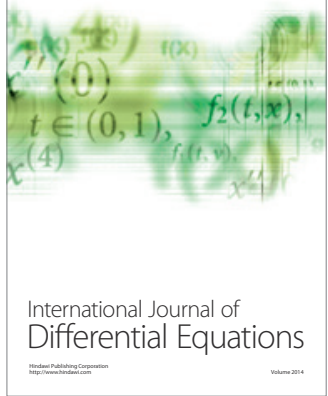
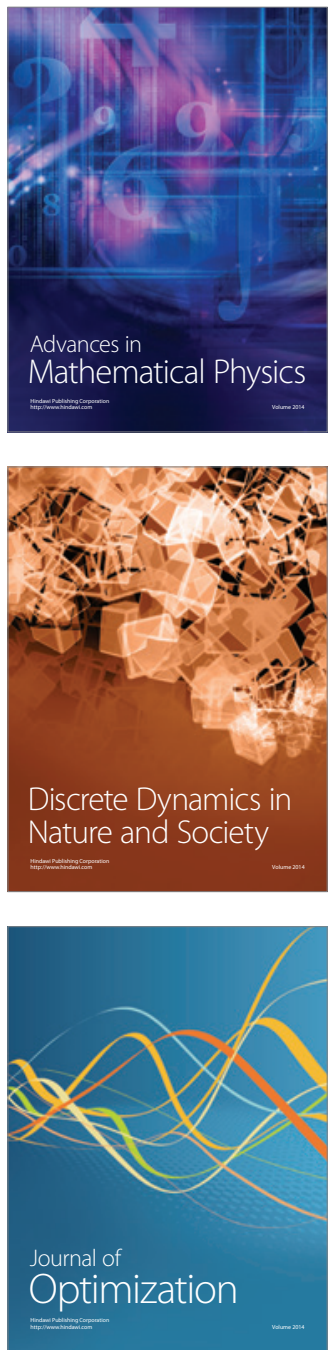